\title{
Some remarks on finiteness of extremal rays of divisorial type
}

\author{
By Yoshio Fujimoto \\ Department of Mathematics, Faculty of Liberal Arts and Sciences, Nara Medical University, \\ 840, Shijo-cho, Kashihara, Nara 634-8521, Japan \\ (Communicated by Shigefumi Mori, M.J.A., Dec. 13, 2021)
}

\begin{abstract}
Let $X$ be a normal Q-factorial projective variety with at most log canonical singularities. We shall give a sufficient condition for the existence of at most finitely many $K_{X}$-negative extremal rays $R(\subset \overline{\mathrm{NE}}(X))$ of divisorial type. As an application, we show that for a nonisomorphic surjective endomorphism $f: X \rightarrow X$ of a normal projective Q-factorial terminal 3-fold $X$ with $\kappa(X)>0$, a suitable power $f^{k}(k>0)$ of $f$ descends to a nonisomorphic surjective endomorphism $g: X_{\min } \rightarrow X_{\min }$ of a minimal model $X_{\min }$ of $X$.
\end{abstract}

Key words: Endomorphism; extremal ray; termination; divisorial contraction; flip.

1. Introduction. The main purpose of this note is to give the following theorem concerning finiteness of extremal rays of divisorial type on a normal projective variety with at most log canonical singularities.

Theorem 1.1. Let $X$ be a normal $\mathbf{Q}$-factorial projective variety with at most log canonical singularities. Suppose that there exists an effective divisor $D$ on $X$ such that for any $K_{X}$-negative extremal ray $R(\subset \overline{\mathrm{NE}}(X))$ of divisorial type, the exceptional divisor $E_{R}$ of the contraction morphism $\operatorname{Cont}_{R}: X \rightarrow X^{\prime}$ is contained in $\operatorname{Supp}(D)$. Then there exist at most finitely many $K_{X}$-negative extremal rays $R$ of divisorial type.

Corollary 1.2. Let $X$ be a normal $\mathbf{Q}$-factorial projective variety with at most canonical singularities. Suppose that $\kappa(X) \geq 0$. Then there exist at most finitely many $K_{X}$-negative extremal rays $R(\subset \overline{\mathrm{NE}}(X))$ of divisorial type.

Let us explain briefly our motivations. Let $f: X \rightarrow X$ be a nonisomorphic étale endomorphism of a normal projective variety $X$ with only canonical singularities. Then it is not necessarily true that for a $K_{X}$-negative extremal ray $R(\subset \overline{\mathrm{NE}}(X))$, there exists a positive integer $k$ such that $\left(f^{k}\right)_{*}(R)=R$ for the automorphism $\left(f^{k}\right)_{*}: \mathrm{N}_{1}(\mathrm{X}) \simeq \mathrm{N}_{1}(\mathrm{X})$ induced from the $k$-th power $f^{k}=f \circ \cdots \circ f$. Thus, if we apply the minimal model program (MMP, for short, cf. [6], [7]) to the study of nonisomorphic surjective endomorphisms of projective varieties, this phe-

2010 Mathematics Subject Classification. Primary 14J15, 14J25, 14J30, 14J60; Secondary 32J17. nomenon causes serious troubles. We cannot always apply the MMP working compatibly with étale endomorphisms. Thus it is an interesting problem to give a sufficient condition for a $K_{X}$-negative extremal ray $R$ to be preserved under a suitable power of $f$. For example, if there exist at most finitely many $K_{X}$-negative extremal rays of divisorial type, then by replacing $f$ by its suitable power $f^{k}(k>0)$, we can apply the MMP working compatibly with nonisomorphic surjective endomorphisms (cf. [1], [2]).

2. Notations and preliminaries. In this paper, we work over the complex number field C. A projective variety is a complex variety embedded in a projective space. By an endomorphism $f: X \rightarrow$ $X$, we mean a morphism from a projective variety $X$ to itself.

The following symbols are used for a variety $X$. $K_{X}$ : the canonical divisor of $X$.

$\operatorname{Aut}(X)$ : the algebraic group of automorphisms of $X$.

$N_{1}(X):=(\{1$-cycles on $X\} / \equiv) \otimes_{\mathbf{z}} \mathbf{R}$, where $\equiv$ means a numerical equivalence.

$N^{1}(X):=(\{$ Cartier divisors on $X\} / \equiv) \otimes_{\mathbf{Z}} \mathbf{R}$, where $\equiv$ means a numerical equivalence.

$\mathrm{NE}(X)$ : the smallest convex cone in $N_{1}(X)$ containing all effective 1-cycles.

$\overline{\mathrm{NE}}(X)$ : the Kleiman-Mori cone of $X$, i.e., the closure of $\mathrm{NE}(X)$ in $N_{1}(X)$ for the metric topology.

$\rho(X):=\operatorname{dim}_{\mathbf{R}} N_{1}(X)$, the Picard number of $X$. $[C]$ : the numerical equivalence class of a 1-cycle C. 
$\operatorname{cl}(D)$ : the numerical equivalence class of a Cartier divisor $D$. $X$.

$\sim_{\mathbf{Q}}$ : the $\mathbf{Q}$-linear equivalence of $\mathbf{Q}$-divisors of

For an endomorphism $f: X \rightarrow X$ and an integer $k>0, f^{k}$ stands for the $k$-times composite $f \circ \cdots \circ f$ of $f$.

Extremal rays: For a normal projective Q-factorial variety $X$ with at most $\log$ canonical singularities, an extremal ray $R$ means a $K_{X}$-negative extremal ray of $\overline{\mathrm{NE}}(X)$, i.e., a 1-dimensional face of $\overline{\mathrm{NE}}(X)$ with $K_{X} R<0$. An extremal ray $R$ defines a proper surjective morphism $\pi_{R}:=$ Cont $_{R}: X \rightarrow Y$ with connected fibers such that, for an irreducible curve $C \subset X, \pi_{R}(C)$ is a point if and only if $[C] \in R$ (cf. [3]). This is called the contraction morphism associated to $R$. If $\pi_{R}$ is birational and contracts a divisor, then $\pi_{R}$ is called a divisorial contraction and $R$ is called of divisorial type. In this case, the exceptional set $\operatorname{Exc}\left(\pi_{R}\right)$ of $\pi_{R}$ is a prime divisor and we denote it by $E_{R}$. If $\pi_{R}$ is birational and $\operatorname{Exc}\left(\pi_{R}\right)$ has codimension $\geq 2$ (i.e., $\pi_{R}$ is small), then $\pi_{R}$ is called a flipping contraction and $R$ is called of flipping type.

For more details and terminologies of the minimal model program, the reader can consult [6] or [7].

3. Proof of Theorem 1.1. We shall give a proof of Theorem 1.1.

Proof of Theorem 1.1. We set $D=\sum_{i=1}^{k} a_{i} D_{i}$, where each $a_{i}$ is a positive integer and each $D_{i}$ is a prime divisor such that $D_{i} \neq D_{j}$ for any $i \neq j$. Suppose that there exist infinitely many $K_{X}$-negative extremal rays $R(\subset \overline{\mathrm{NE}}(X))$ of divisorial type and we shall derive a contradiction. We follow the idea of the proof of [9, Lemma 6.2]. Then there exists a prime divisor $D_{i}$ such that $D_{i}=E_{R}$ for infinitely many extremal rays $R$ of divisorial type. Let $\mathcal{S}$ be an infinite set consisting of extremal rays $R(\subset \overline{\mathrm{NE}}(X))$ such that $E_{R}=D_{i}$. For $R \in \mathcal{S}$, let $\pi_{R}:=\operatorname{Cont}_{R}: X \rightarrow Y_{R}$ be the divisorial contraction morphism associated to $R$. We set $N_{\mathbf{C}}^{1}(X):=$ $N^{1}(X) \otimes_{\mathbf{R}}$ C. We have the following commutative diagram

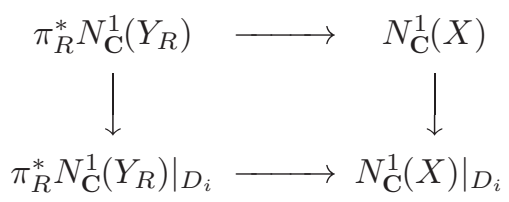

where both horizontal arrows are inclusions and both vertical arrows are surjections. Then by the cone theorem (cf. [3], [4], [6], [7]), $\pi_{R}^{*} N_{\mathbf{C}}^{1}\left(Y_{R}\right) \hookrightarrow$ $N_{\mathbf{C}}^{1}(X)$ is a linear subspace of codimension one. Hence $\Delta_{R}:=\left.\left.\pi_{R}^{*} N_{\mathbf{C}}^{1}\left(Y_{R}\right)\right|_{D_{i}} \hookrightarrow N_{\mathbf{C}}^{1}(X)\right|_{D_{i}}$ is also a linear subspace of codimension at most one. On the other hand, $\left.H\right|_{D_{i}}$ is not contained in $\Delta_{R}$ for an ample divisor $H$ of $X$. Hence $\Delta_{R}$ is of codimension one in $\left.N_{\mathbf{C}}^{1}(X)\right|_{D_{i}}$. If we set $V:=\{v \in$ $\left.\left.N_{\mathbf{C}}^{1}(X)\right|_{D_{i}} ; v^{\operatorname{dim} X-1}=0\right\}$, then $V$ is an affine hypersurface of degree $\operatorname{dim} X-1$ in the complex vector space $\left.N_{\mathbf{C}}^{1}(X)\right|_{D_{i}}$. Since $\operatorname{dim} \pi_{R}\left(D_{i}\right) \leq \operatorname{dim} X-2$, the complex vector space $\Delta_{R}$ is contained in $V$. Since $\operatorname{dim} V=\operatorname{dim} \Delta_{R}=\operatorname{dim}\left(\left.N_{\mathbf{C}}^{1}(X)\right|_{D_{i}}\right)-1, \Delta_{R}$ is an irreducible component of $V$. Let $C_{R}$ be an extremal curve on $X$ whose numerical class $\left[C_{R}\right]$ spans $R$. Then $\left[C_{R}\right]$ is orthogonal to $\Delta_{R}$ via the intersection pairing. If $R \neq R^{\prime} \in \mathcal{S}$, then $C_{R}$ is not contracted to a point by $\pi_{R^{\prime}}$ and $\left[C_{R}\right]$ is not orthogonal to $\Delta_{R^{\prime}}$. Hence $\Delta_{R} \neq \Delta_{R^{\prime}}$ and $V$ has an infinite number of irreducible components $\Delta_{R}(R \in \mathcal{S})$. Since the number of all the irreducible components of $V$ is finite, this is a contradiction. Thus the proof is finished.

Proof of Corollary 1.2. Since $\kappa(X) \geq 0, m K_{X}$ is a Cartier divisor and $\left|m K_{X}\right| \neq \emptyset$ for some positive integer $m$. Take a member $D \in\left|m K_{X}\right|$ and we set $D=\sum_{i=1}^{k} a_{i} D_{i}$, where each $a_{i}$ is a positive integer and each $D_{i}$ is a prime divisor such that $D_{i} \neq D_{j}$ for any $i \neq j$. For any $K_{X}$-negative extremal ray $R$ of divisorial type, take an extremal curve $C_{R}$ whose numerical class $\left[C_{R}\right]$ spans $R$. Since $0>$ $m\left(K_{X}, C_{R}\right)=\sum_{i} a_{i}\left(D_{i}, C_{R}\right)$, we have $\left(D_{i}, C_{R}\right)<0$ for some $i$. Hence $C_{R}$ is contained in $D_{i}$. Since $C_{R}$ sweeps out $E_{R}$, we have $E_{R} \subset D_{i}$. Hence $E_{R}=D_{i}$, since $D_{i}$ is irreducible. Then applying Theorem 1.1 to $D$, the proof follows immediately.

Next, we shall consider extremal rays of an almost homogeneous variety.

Definition 3.1. Let $X$ be an irreducible normal algebraic variety. Suppose that a connected algebraic group $G$ acts algebraically on $X$. If the group $G$ has an open dense orbit in $X$, then $X$ is called almost homogeneous (with respect to the action of $G$ ), or the $G$-action on $X$ is almost transitive. In particular, if $\operatorname{Aut}^{0}(X)$ has an open dense orbit in $X$, then we say that $X$ is almost homogeneous.

Corollary 3.2. Let $G$ be a connected positive dimensional algebraic group which acts regularly on a smooth projective variety X. Suppose that 
$X$ is almost homogeneous with respect to the G-action (cf. Definition 3.1). Then the number of $K_{X}$-negative extremal rays of divisorial type on $\overline{\mathrm{NE}}(X)$ is finite.

Proof. Let $X^{0}$ be an open dense orbit of $G$ and $S:=X \backslash X^{0}$ its complement. For any extremal ray $R$ of divisorial type, let $E_{R}$ be the exceptional divisor of the contraction morphism Cont $_{R}$ associated to $R$. First we show that $E_{R} \subset S$. The proof is by contradiction. Assume the contrary. Then, there exists some point $P \in E_{R} \cap X^{0}$. Let $\ell$ be an extremal rational curve on $X$ which passes through $P$ and its numerical class $[\ell]$ spans $R$. By assumption, for any $Q \in X^{0}$ there exists some $g \in G$ such that $g(P)=Q$. Since $G$ is connected, it acts trivially on the homology group $H_{2}(X, \mathbf{Z})$ which is discrete, and hence on $H_{2}(X, \mathbf{R})$. Thus the action of $G$ on $\overline{\mathrm{NE}}(X)$ is also trivial. Hence $g(\ell)$ is an extremal rational curve passing through $Q$ and its numerical class $[g(\ell)]=[\ell]$ also spans the same extremal ray $R$. Thus $Q(\in g(\ell))$ is contained in $E_{R}$. Hence the open dense $G$-orbit $X^{0}$ is contained in the exceptional divisor $E_{R}$, which derives a contradiction. Let $D$ be a reduced divisor on $X$ which is a sum of all the prime divisors contained in $S$. Then $E_{R} \subset \operatorname{Supp}(D)$ for any $K_{X}$-negative extremal ray $R$ of divisorial type. Hence applied Theorem 1.1, we see that the number of all the $K_{X}$-negative extremal rays $R$ of divisorial type is finite.

4. Applications to endomorphisms. In this section, as an application of Therem 1.1, we shall apply the MMP to a nonisomorphic surjective endomorphism $f: X \rightarrow X$ of a normal Q-factorial projective 3 -fold $X$ with only terminal singularities and $\kappa(X)>0$. We recall the following fundamental result.

Lemma 4.1. Let $f: X \rightarrow X$ be a surjective endomorphism of a normal $\mathbf{Q}$-factorial projective variety $X$. Suppose that $K_{X}$ is pseudo-effective. Then $f$ is a finite morphism which is étale in codimension one.

Proof. The proof follows immediately by the same argument as in the proof of [1, Lemma 2.3].

Lemma 4.2 (cf. $\quad[1, \quad$ Propositions 4.2 and 4.12]). Let $f: Y \rightarrow X$ be a surjective morphism between normal, Q-factorial projective log canonical $n$-folds with $\rho(X)=\rho(Y)$. Then the following hold.

(1) $f$ is a finite morphism and the push-forward map $f_{*}: N_{1}(Y) \rightarrow N_{1}(X)$ is an isomorphism and $f_{*} \overline{\mathrm{NE}}(Y)=\overline{\mathrm{NE}}(X)$.

(2) Let $f_{*}: N^{1}(Y) \rightarrow N^{1}(X)$ be the map induced from the push-forward map $D \mapsto f_{*} D$ of divisors. Then the dual map $f^{*}: N_{1}(X) \rightarrow N_{1}(Y)$ is an isomorphism and $f^{*} \overline{\mathrm{NE}}(X)=\overline{\mathrm{NE}}(Y)$.

(3) If $f$ is étale in codimension one and $K_{X}$ is not nef, then $f^{*}$ and $f_{*}$ above give a one-to-one correspondence between the set of extremal rays of $X$ and $Y$.

(4) Under the same assumption as in (3), for an extremal ray $R(\subset \overline{\mathrm{NE}}(Y))$, and for the contraction morphisms $\operatorname{Cont}_{R}: Y \rightarrow Y^{\prime}$ and $\operatorname{Cont}_{f_{*} R}: X \rightarrow X^{\prime}$, there exists a finite surjective morphism $f^{\prime}: Y^{\prime} \rightarrow X^{\prime}$ such that $f^{\prime} \circ \operatorname{Cont}_{R}=$ Cont $_{f_{*} R} \circ f$.

Proof. Since the cone and contraction theorem holds if $X$ is a $\mathbf{Q}$-factorial $\log$ canonical $n$-fold (cf. [3]), the proof follows immediately by the same argument as in the proof of [1, Propositions 4.2 and 4.12$]$.

Lemma 4.3. Let $f: X \rightarrow X$ be a nonisomorphic surjective endomorphism of a normal, Q-factorial projective $n$-fold $X$ with only canonical singularities and $\kappa(X) \geq 0$. Suppose that $K_{X}$ is not nef and there exists a $K_{X}$-negative extremal ray $R(\subset \overline{\mathrm{NE}}(X))$ of divisorial type. Then replacing $f$ by its suitable power $f^{k}(k>0)$, there exits the following commutative diagram

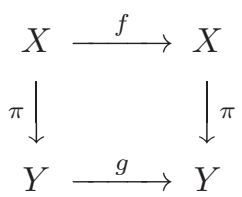

which is almost Cartesian (i.e., the fiber product when restricted over a Zariski open subset $Y^{0}$ of $Y)$ such that the following hold:

(1) $\pi: X \rightarrow Y$ is an extremal divisorial contraction associated to $R$ and contracts a prime divisor on $X$ to a positive-dimensional subvariety on $Y$.

(2) $g: Y \rightarrow Y$ is a a nonisomorphic surjective endomorphism of a $\mathbf{Q}$-factorial variety $Y$ with at most canonical singularities.

Proof. Let $\pi:=\operatorname{Cont}_{R}: X \rightarrow Y$ be an extremal divisorial contraction associated to $R$. By Corollary 1.2 we see that $\left(f^{k}\right)_{*} R=R$ for some integer $k>0$. Hence, if we replace $f$ by its power $f^{k}$ and applied Lemma $4.2, f$ descends to a nonisomorphic surjective endomorphism $g$ of $Y$. By Lemma 4.2, we see that $g$ is finite and étale in codimension one. If we set $E:=\operatorname{Exc}(\pi)$, then $E$ is a prime divisor on $X$ and 
$K_{X} \sim_{\mathrm{Q}} \pi^{*} K_{Y}+a E$ for a positive rational number $a>0$. We have $K_{X} \sim_{\mathbf{Q}} f^{*} K_{X}$ and $K_{Y} \sim_{\mathbf{Q}} g^{*} K_{Y}$, since both $f$ and $g$ are finite morphisms étale in codimension one. Since $\pi \circ f=g \circ \pi$, we have $f^{*} E \sim_{\mathbf{Q}} E$. Suppose that $\pi(E)$ is a point on $Y$. Since $-E$ is $\pi$-ample, we have $\left(-\left.E\right|_{E}\right)^{(n-1)}>0$. Since $\left(\left.f\right|_{E}\right)^{*}\left(-\left.E\right|_{E}\right) \sim_{\mathbf{Q}}-\left.E\right|_{E}$, we have $\left(-\left.E\right|_{E}\right)^{n-1}=$ $\operatorname{deg}\left(\left.f\right|_{E}\right)\left(-\left.E\right|_{E}\right)^{n-1}$. Then we have $\left(-\left.E\right|_{E}\right)^{n-1}=0$, since $\operatorname{deg}\left(\left.f\right|_{E}\right)=\operatorname{deg}(f) \geq 2$. Thus a contradiction is derived and $\pi(E)$ is not a point on $Y$.

Proposition 4.4. Let $f: X \rightarrow X$ be a nonisomorphic surjective endomorphism of a normal Q-factorial projective variety $X$ with at most canonical singularities. Suppose that $\kappa(X) \geq 0$ and $K_{X}$ is not nef. Then replacing $f$ by its suitable power $f^{k}(k>0)$, there exits the following finite sequence of birational morphisms

$$
X=X_{1} \stackrel{\pi_{1}}{\longrightarrow} \cdots \rightarrow X_{i} \stackrel{\pi_{i}}{\longrightarrow} X_{i+1} \rightarrow \cdots \rightarrow X_{k}=Y
$$

such that

(1) each $\pi_{i}$ is an extremal divisorial contraction which contracts a prime divisor $E_{i}$ on $X_{i}$ to a positive-dimensional subvariety on $X_{i+1}$,

(2) $f=f_{1}$ descends to a nonisomorphic surjective endomorphism $f_{i}: X_{i} \rightarrow X_{i}$ of a Q-factorial normal projective variety $X_{i}$ with at most canonical singularities, and

(3) any $K_{Y}$-negative extremal ray $R(\subset \overline{\mathrm{NE}}(Y))$ is of flipping type, i.e., the contraction morphism associated to $R$ is small.

Proof. We may assume that there exists some $K_{X}$-negative extremal ray $R_{1}\left(\subset \overline{\mathrm{NE}}\left(X_{1}\right)\right)$ of divisorial type. Let $\pi_{1}: X=X_{1} \rightarrow X_{2}$ be the extremal divisorial contraction associated to $R_{1}$. Then Lemma 4.3 shows that if we replace $f$ by its suitable power $f^{\ell}(\ell>0)$, then $f$ descends to a nonisomorphic surjective endomorphism $f_{2}: X_{2} \rightarrow X_{2}$ of $X_{2}$. If there exists some $K_{X_{2}}$-negative extremal ray $R_{2}\left(\subset \overline{\mathrm{NE}}\left(X_{2}\right)\right)$ of divisorial type, then we repeat the same procedure and obtain the following sequence

$$
X=X_{1} \stackrel{\pi_{1}}{\longrightarrow} X_{2} \stackrel{\pi_{2}}{\longrightarrow} \cdots \rightarrow X_{i} \stackrel{\pi_{i}}{\longrightarrow} X_{i+1} \rightarrow \cdots \rightarrow \cdots,
$$

where

- each $\pi_{i}$ is an extremal divisorial contraction which contracts a prime divisor on $X_{i}$ to a positive-dimensional subvariety on $X_{i+1}$, and

- $f$ descends to a nonisomorphic surjective endomorphism $f_{i}: X_{i} \rightarrow X_{i}$ of $X_{i}$.

Since $\rho\left(X_{i+1}\right)=\rho\left(X_{i}\right)-1$, these procedures eventually stop. Hence there exists no $K_{X_{k}}$-negative extremal ray of divisorial type for some $k>0$ and we set $Y:=X_{k}$. Then any $K_{Y}$-negative extremal ray $R(\subset \overline{\mathrm{NE}}(Y))$ is of flipping type and we are done.

Remark 4.5 (cf. [1, Theorem 4.8, Proposition 4.9, and Definition 4.15]). Let $f: X \rightarrow X$ be a nonisomorphic surjective endomorphism of a smooth projective 3 -fold $X$ with $\kappa(X) \geq 0$. Then, for any $i, X_{i}$ is nonsingular and $\pi_{i-1}: X_{i-1} \rightarrow X_{i}$ is the blowing-up of an elliptic curve $C_{i}\left(\subset X_{i}\right)$ such that $f_{i}^{-1}\left(C_{i}\right)=C_{i}$. Note that there exists no $K_{Y}$-negative extremal ray of flipping type on $\overline{\mathrm{NE}}(Y)$, since $Y$ is a smooth projective 3 -fold (cf. [10]). In this case, $Y=X_{k}$ is the unique minimal model of $X$ and $f_{k}: Y \rightarrow Y$ is called the minimal reduction of $f: X \rightarrow X$.

Next, we shall apply the MMP to a nonisomorphic surjective endomorphism $f: X \rightarrow X$ of a normal projective $\mathbf{Q}$-factorial terminal 3 -fold $X$ with $\kappa(X)>0$.

Theorem 4.6. Let $f: X \rightarrow X$ be a nonisomorphic surjective endomorphism of a normal projective Q-factorial 3-fold $X$ with only terminal singularities. Suppose that $\kappa(X)>0$. Then if we replace $f$ by its suitable power $f^{k}(k>0)$, there exists the following commutative diagram

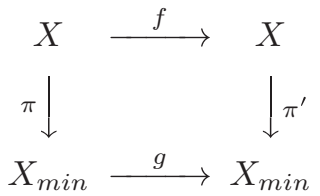

which satisfies the following

(1) $X_{\min }$ is a minimal model of $X$, i.e., $X_{\min }$ is a normal, projective $\mathbf{Q}$-factorial terminal 3-fold which is birational to $X$ and $K_{X_{\min }}$ is nef.

(2) $\pi^{\prime}$ is a composition of a finite number of divisorial contractions contracting a prime divisor to a curve, and a finite number of terminal flips.

(3) $\pi=w \circ \mu$, where $\mu: X \cdots \rightarrow X^{\prime}$ is a composition of a finite number of divisorial contractions contracting a prime divisor to a curve and a finite number of terminal flips, and $w: X^{\prime} \simeq X_{\min }$ is an isomorphism.

(4) $g$ is a nonisomorphic surjective endomorphism of $X_{\min }$.

Proof. We may assume that $K_{X}$ is not nef. Then applied Proposition 4.4 and replacing $f$ by its suitable power $f^{k}(k>0)$, there exits the following commutative diagram 


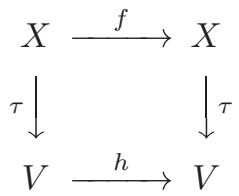

which is almost Cartesian (i.e., the fiber product when restricted over a Zariski open subset $V^{0}$ of $V$ ) such that the following hold:

(1) $\tau: X \rightarrow V$ is a succession of extremal divisorial contractions which contracts a prime divisor to a curve.

(2) $h: V \rightarrow V$ is a nonisomorphic surjective endomorphism of a normal Q-factorial projective 3fold $V$ with only terminal singularities.

(3) Any $K_{V}$-negative extremal ray $R(\subset \overline{\mathrm{NE}}(V))$ is of flipping type.

Hereafter, we may assume that $K_{V}$ is not nef. Take a $K_{V}$-negative extreal ray $R^{(1)}(\subset \overline{\mathrm{NE}}(V))$. We set $R_{0}^{(1)}:=R^{(1)}$ and $R_{n}^{(1)}:=\left(f^{n}\right)_{*}\left(R^{(1)}\right), R_{-n}^{(1)}:=$ $\left(f^{n}\right)^{*} R^{(1)}$ for a positive integer $n$. Then by Lemma 4.2 , we see that $R_{n}^{(1)}(\subset \overline{\mathrm{NE}}(V))$ is a $K_{V}$-negative extremal ray of flipping type for any $n \in \mathbf{Z}$. Let $u_{n}: V \rightarrow W_{n}$ be the small birational contraction associated to $R_{n}^{(1)}$. Then for any $n \in \mathbf{Z}$, there exits the following commutative diagram

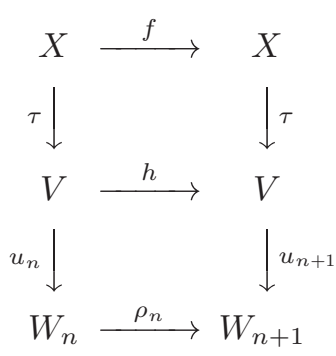

where $\rho_{n}$ is a nonisomorphic finite morphism étale in codimension one. The first (resp. the second) commutative diagram from the top is almost Cartesian, i.e., the fiber product when restricted over a Zariski open subset $V^{0}$ of $V$ (resp. $W_{n+1}^{0}$ of $\left.W_{n+1}\right)$. Then by [11], the canonical $\operatorname{ring} R_{n}:=$ $\oplus_{m \geq 0} u_{n *}\left(\mathcal{O}_{V}\left(m K_{V}\right)\right)$ is a finitely generated $\mathcal{O}_{W_{n}}$-algebra and set $V_{n}^{+}:=\operatorname{Proj}_{W_{n}}\left(R_{n}\right)$. Then $u_{n}^{+}: V_{n}^{+} \rightarrow$ $W_{n}$ is a flip of $u_{n}: V \rightarrow W_{n}$. Let $U_{n}$ be the normalization of $V_{n+1}^{+} \times_{W_{n+1}} W_{n}$. Then $K_{U_{n}}$ is a well-defined Q-Cartier divisor since $U_{n} \rightarrow V_{n+1}^{+}$is finite and étale in codimension one. Note that $K_{U_{n}}$ is the pull-back of $K_{V_{n+1}^{+}}$by construction. Therefore, $K_{U_{n}}$ is ample over $\stackrel{n+1}{W}_{n}$ and $U_{n} \rightarrow W_{n}$ is small by construction. Hence $U_{n}$ is a flip of $V \rightarrow W_{n}$ and $U_{n} \simeq V_{n}^{+}$(cf. [7, Lemma 6.2]). By this observation, for any $n \in \mathbf{Z}$, we can construct the commutative diagram of flip

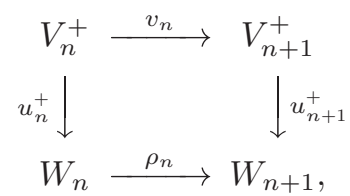

where $V_{n}^{+}$is a normal $\mathbf{Q}$-factorial projective 3 -fold with only terminal singularities and the natural projection $v_{n}$ is a nonisomorphic finite morphism which is étale in codimension one. If $K_{V_{n}^{+}}$is nef, then we stop. If $K_{V_{n}^{+}}$is not nef, then we repeat the same procedure. Because of the termination of 3-fold flips (cf. [11]), these procedures eventually stop after finitely many times and for any $n \in \mathbf{Z}$, we obtain the following commutative diagram

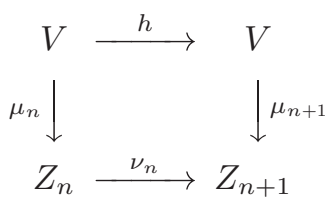

which satisfies the following

(1) $Z_{n}$ is a minimal model of $V$ (hence of $X$ ), i.e., $K_{Z_{n}}$ is nef.

(2) $\mu_{n}$ is a composition of finitely many terminal flips.

(3) $\nu_{n}$ is a nonisomorphic finite morphism which is étale in codimension one.

Since $\kappa(X)>0,[5$, Theorem 4.5] shows that there exist only finitely many minimal models of $X$ up to isomorphisms. Hence there exists an isomorphism $w: Z_{p} \simeq Z_{q}$ for some integers $p<q$. Thus we have the following commutative diagram

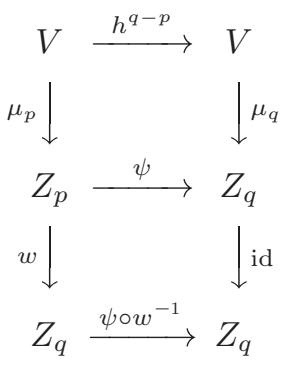

where we set $\psi:=\nu_{q-1} \circ \cdots \nu_{p}$. Hence if we further replace $f$ (resp. $h$ ) by its positive power $f^{q-p}$ (resp. $\left.h^{q-p}\right)$ and set $X_{\min }:=Z_{q}, X^{\prime}:=Z_{p}, v=w \circ \mu_{p}, v^{\prime}=$ $\mu_{q}$, and $g:=\psi \circ w^{-1}$, then we obtain the following commutative diagram 


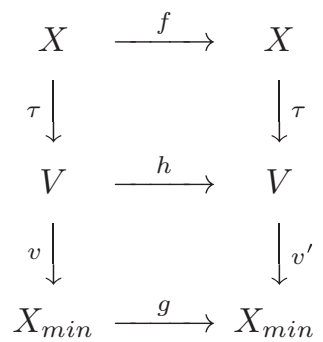

which satisfies the following

(1) $\tau$ is a succession of extremal divisorial contractions which contracts a prime divisor to a curve.

(2) $v=w \circ \mu_{p}$, where $\mu_{p}: X \cdots \rightarrow X^{\prime}$ is a composition of finitely many terminal flips and $w$ : $X^{\prime} \simeq X_{\min }$ is an isomorphism.

(3) $v^{\prime}$ is a composition of finitely many terminal flips.

(4) $g$ is a nonisomorphic surjective endomorphism of $X_{\min }$.

Thus if we set $\mu:=\mu_{p} \circ \tau: X \cdots \rightarrow X^{\prime}, \pi:=w \circ$ $\mu(=v \circ \tau)$ and $\pi^{\prime}:=v^{\prime} \circ \tau$, then the proof is finished.

Remark 4.7. (1) In [5], the finiteness of minimal models of $X$ is not established in the case of $\kappa(X)=0$. Thus by the proof of Theorem 4.6, we can show the following

'Suppose that $\kappa(X)=0$ in the assumption of Theorem 4.6. Then, after a finite number of divisorial contractions and terminal flips, an endomorphism $f: X \rightarrow X$ induces a tower of nonisomorphic finite morphisms $\left\{Z_{n} \rightarrow\right.$ $\left.Z_{n+1}\right\}_{n \in \mathbf{Z}}$ between minimal models $Z_{n}$ of $X$ which is étale in codimension one.'

(2) The conclusion of Lemma 4.3 does not necessarily hold for a $K_{X}$-negative extremal ray $R(\subset \overline{\mathrm{NE}}(X))$ of flipping type. We shall give such an example. [8, Theorem 7.1] shows the existence of a terminal, projective 3 -fold $Y$ of nonnegative Kodaira dimension with infinitely many $K_{Y}$-negative extremal rays of flipping type. $Y$ has a fiber space structure $\varphi: Y \rightarrow \Gamma$ over a curve $\Gamma$ of genus $g(\Gamma) \geq 1$ whose general fiber is isomorphic to the product $E \times E$ of an elliptic curve $E$. Moreover, a $K_{Y}$-negative flipping curve $\ell$ is contained in a fiber of $\varphi: Y \rightarrow \Gamma$. The relative automorphism group $\operatorname{Aut}(Y / \Gamma)$ of $Y$ over $\Gamma$ contains a subgroup $G$ which is isomorphic to $\operatorname{SL}(2, \mathbf{Z})$. The $G$-orbit of $\ell$ all give $K_{Y}$-negative extremal curves of flipping type. Let $C$ be an elliptic curve and $\mu_{n}: C \rightarrow C$ be a multiplication mapping by a positive integer $n>1$. We take an element $g \in G$ of infinite order. Let $X:=Y \times C$ be the product of $Y$ and $C$. Then $\tau:=g \times \mu_{n}: X \rightarrow X$ gives a nonisomorphic surjective endomorphism of a terminal 4-fold $X$ with $\kappa(X)=$ $\kappa(Y) \geq 0$. The numerical class $[\gamma]$ of a curve $\gamma:=\ell \times\{o\}(o \in C)$ also spans a $K_{X}$-negative extremal ray $L(\subset \overline{\mathrm{NE}}(X))$ of flipping type. By construction, $\left(\tau^{k}\right)_{*} L \neq L$ for any positive integer $k>0$.

Acknowledgements. The author wishes to express sincere thanks to the referee for the careful reading of the manuscript and error corrections. The author is supported by the Grant-in-Aid for Scientific research (C), Japan Society for the Promotion of Science.

\section{References}

[ 1 ] Y. Fujimoto, Endomorphisms of smooth projective 3-folds with non-negative Kodaira dimension, Publ. Res. Inst. Math. Sci. 38 (2002), no. $1,33-92$.

[ 2 ] Y. Fujimoto, Étale endomorphisms of 3-folds. I, Osaka J. Math. 55 (2018), no. 2, 195-257.

[ 3 ] O. Fujino, Fundamental theorems for the log minimal model program, Publ. Res. Inst. Math. Sci. 47 (2011), no. 3, 727-789.

[ 4 ] Y. Kawamata, The cone of curves of algebraic varieties, Ann. of Math. (2) 119 (1984), no. 3, 603-633.

[ 5 ] Y. Kawamata, On the cone of divisors of CalabiYau fiber spaces, Internat. J. Math. 8 (1997), no. $5,665-687$.

[6 ] Y. Kawamata, K. Matsuda and K. Matsuki, Introduction to the minimal model problem, in Algebraic geometry, Sendai, 1985, Adv. Stud. Pure Math., 10, North-Holland, Amsterdam, 1987, pp. 283-360.

[ 7 ] J. Kollár and S. Mori, Birational geometry of algebraic varieties, translated from the 1998 Japanese original, Cambridge Tracts in Mathematics, 134, Cambridge University Press, Cambridge, 1998.

[ 8 ] J. Lesieutre, Some constraints of positive entropy automorphisms of smooth threefolds, Ann. Sci. Éc. Norm. Supér. (4) 51 (2018), no. 6, 15071547.

[ 9 ] S. Meng and D.-Q. Zhang, Building blocks of polarized endomorphisms of normal projective varieties, Adv. Math. 325 (2018), 243-273.

[10] S. Mori, Threefolds whose canonical bundles are not numerically effective, Ann. of Math. (2) 116 (1982), no. 1, 133-176.

[11] S. Mori, Flip theorem and the existence of minimal models for 3-folds, J. Amer. Math. Soc. 1 (1988), no. 1, 117-253. 